\title{
Hypothesis: Mycotoxins Causing Amyotrophic Lateral Sclerosis
}

\author{
William Reid ${ }^{1}$ \\ ${ }^{1}$ University of North Carolina at Chapel Hill
}

August 17, 2020

\begin{abstract}
Amyotrophic Lateral Sclerosis(ALS) remains a terminal disease without an established etiology for the majority of patients. The dominant theory of ALS before 1970's was the presence of a poison. One of the primary means of treating patients with a toxic exposure has been plasma exchange, but plasma exchange of ALS patients failed to alter the clinical course. The failure of plasma exchange assumes the patient is no longer exposed to the poison. If the exposure to poisons continued, then plasma exchange alone would fail. I found laboratory evidence of a poisoning in every patient with ALS examined. A search for specific poisons found evidence of mycotoxins. Treatment with antifungal agents corrected the laboratory findings. All of the ALS patients had evidence of immune suppression. There is mounting evidence that many mycotoxins cause both neurotoxicity and immune suppression. These mycotoxins may be able to explain the full spectrum of pathology in ALS without a secondary event.
\end{abstract}

\section{Introduction :}

The etiology of ALS has remained elusive. The dominant theory before the 1970's was the presence of neurotoxins ${ }^{1,2}$. Serum from patients with ALS were toxic to motor neuron cells in culture. Based on this theory multiple institutions treated ALS patients with plasma exchange without apparent improvement ${ }^{3}$. The conclusion that plasma exchange was ineffective made a critical assumption that there is a finite body burden of neurotoxin and no further exposures. If, instead exposure to neurotoxins persist after plasma exchange then plasma exchange would be inadequate. The source of toxins whether environmental or endogenous would need to be removed. If neurotoxins were generated by an infectious agent colonizing or infecting patients, then treatment would require control of the infection along with plasma exchange.

In every ALS patient studied, there was laboratory evidence of poisons ${ }^{4}$. Patients developed secondary porphyria in a pattern typical of poisoning. They developed anion-gap positive metabolic acidosis. They all had elevated Kreb cycle intermediates consistent with mitochondrial damage typically seen with toxic exposures. Treatment with anti-fungal agents corrected all the laboratory findings including the elevated protoporphyrins, the anion-gap positive metabolic acidosis and the elevated Kreb cycle intermediates consistent with the presence of mycotoxin generated by a fungus growing in the patient.

The literature on mycotoxins is extensive ${ }^{5-7}$. Mycotoxins are ubiquitous in the human environment including the world food supply. Globalization has accentuated the problem. Efforts to prevent or remove fungi and their mycotoxins from food and the environment have been difficult to impossible. Humans are routinely colonized by fungi that populate the skin, gastrointestinal tract, lungs and upper airway ${ }^{8,9}$. The same fungal species contaminating food are the species colonizing the human airways. Surveys of grain supplies found hundreds of fungal species producing over 700 mycotoxins $^{5}$. Four fungal genera were routinely reported Aspergillus, Penicillium, Alternaria and Fusarium ${ }^{10,11}$. Of these fungal genera, Fusarium species were especially problematic ${ }^{12}$. Studies from otolaryngology reported that fungal colonization of the sinus cavity and upper airway is almost ubiquitous ${ }^{13-17}$. These studies found the same spectrum of fungal species with 
Aspergillus and Fusarium listed first and second in frequency. Many of these fungal species are highly resistant to anti-fungal agents especially Fusarium species. Fusarium species produce a broad spectrum of mycotoxins with Fumonisins and Trichothecenes predominate ${ }^{12}$. Both these compounds have significant neurologic toxicity ${ }^{18}$. The problem of contamination of the human food chain by fungi and their mycotoxins has led to a world-wide industry to control the contamination of food (Romer Labs, Vicom Labs division Waters). Mycotoxin levels vary with environmental factors as well as storage techniques. There has been no successful way to prevent or remove these mycotoxins from food. This has left governmental authorities with few options. They have elected to monitor food supplies, set limits of exposure and discard food that exceed limits.

Animals fed hay or grain contaminated with mycotoxins develop serious problems that include neurologic deficits $^{19-21}$. Horses exposed to hay or grain contaminated with Fumonisins develop Equine Leukoencephalomalacia with paralysis of tongue, incoordination, ataxia, blindness, hind limb paralysis and eventual death with pathology finding severe brain damage.

The other dominant mycotoxins from Fusarium are Trichothecenes divided into four groups, A,B,C \& $D^{12}$. The key to their toxicity is the presence of an epoxide ring moiety that generates free radicals setting off Oxidative Stress Reactions. They form covalent bonds to DNA, RNA and proteins. They target the 60S ribosome and peptidyl transferase shutting down protein production. They are highly lipophilic able to be absorbed through skin, gut, crossing plasma membranes, including the blood brain barrier. Trichothecenes are known to accumulate in nervous tissue ${ }^{18}$. In surveys of grain, the trichothecene Deoxynivalenol was the most common with T-2/HT-2 Toxins the fourth most common. Studies of Deoxynivalenol found that it could accumulate in tissues even when levels were below the regulatory limits ${ }^{22}$.

\section{Neurotoxicity \& Pathology of T-2 Toxins mimics ALS Pathology}

A review of T-2/HT-2 Toxins ${ }^{18}$ summarized the mounting evidence of its profound neurologic toxicity. The pathology of T-2 toxin mimics the pathology found in ALS. T-2/ HT-2 toxins are able to be absorbed across skin, mucosa and the blood-brain barrier accumulating in the CNS. They induce reactive oxygen species with Oxidative Stress Reactions. They cause mitochondrial dysfunction and damage to a cascade of signaling pathways including p53, MAPK, Akt/mTOR, PKA/CREB and NF-kB with neuronal cell death. They cause perturbations of the mitochondrial respiratory chain with loss of ATP energy supplies. The motor neurons of the spinal cord have a relatively high demand for ATP.

\section{Immune Suppression Due to Trichothecenes :}

The broad scope of pathology with mycotoxins like T-2/HT-2 Toxin includes damage to lymphocytes leading to humeral and cellular immune deficiency ${ }^{23-26}$. Similar immune deficits are found in ALS with reports of Subclass IgG deficiency ${ }^{27,28}$ and T-cell Immune deficiency. The immune compromise induced by T-2/HT-2 Toxins and other mycotoxins accentuates the proliferation of the fungus leading to eventual fungal invasion, but this may be a late phenomenon.

\section{Revised Hypothesis-Trichothecenes Induced Neurotoxicity does not require Fungal Invasion:}

There is epidemiologic evidence of clusters of ALS in areas with environmental toxicity. The fungal species contaminating the food chain are opportunistic and unable to invade tissue without severe immune suppression. The level of immune suppression required for tissue invasion is equivalent to that seen post bone marrow transplant, especially severe neutropenia. This level of immune suppression is not routinely found in patients with ALS. Mycotoxins can create a localized immune deficit that may allow the fungus to persist while generating neurotoxins that accumulate locally. Fumonisins and Trichothecenes are able to cross plasma membranes including the blood brain barrier and accumulate in the CNS. The immune deficits due to these toxins may become systemic late in the disease. The full scope of pathology generated by these poisons can explain all the neurologic disease without fungal invasion.

Based on this literature, ALS may be due to mycotoxin(s) generated by fungi colonizing the human upper airway, mouth, sinus cavity or even a tooth socket. Any of the hundreds of fungal contaminants could be a 
responsible, but Fusarium species seem especially suspect releasing Fumonisins and Trichothecenes.

Previously, I assumed that patients needed to be immune suppressed before these fungi could infect and release mycotoxins. It now seems possible that opportunistic fungi can colonize the upper airways releasing mycotoxins in immune competent patients. These mycotoxins are highly lipophilic able to cross into the base of the brain and cerebrospinal fluid accumulating in the spinal cord. Fungal invasion is unlikely to be a factor until late in the course of the disease.

\section{Treatment:}

If ALS is due to mycotoxins generated by fungi colonizing patients, then treatment would require antifungal agents. Fusarium species are prime suspects presenting the added problem of resistance to almost all the standard antifungal agents. Voraconazole has shown some promise controlling Fusarium species but treatment might require combinations such as Amphotericin plus Voraconazole. Due to the immune suppression, these patients would require Gammaglobulin IM, Subcutis or IV. Other efforts to restore the immune deficit might require Interleukins or Interferons. Plasma Exchange would be critical to lower the body burden of toxins. There are new options that might improve the removal of mycotoxins using hemodialysis against Charcoal or Affinity Columns. A search for a focus of fungal colonization would be important with an aggressive effort to remove it. Patients may have additional deficits due to pituitary damage. Hypopituitarism could mask a response to treatment.

William K. Reid Date

1. Wolfgram F, Myers L. Amyotrophic lateral sclerosis: effect of serum on anterior horn cells in tissue culture. Science.1973;179(4073):579-580.

2. Field EJ, Hughes D. Toxicity of motor neurone disease serum for myelin in tissue culture. $\mathrm{Br} \mathrm{Med} J$. 1965;2(5475):1399-1401.

3. Silani V, Scarlato G, Valli G, Marconi M. Plasma Exchange Ineffective in Amyotrophic Lateral Sclerosis. Archives of Neurology.1980;37(8):511-513.

4. Reid W. Immunosuppression \& Mycotoxins Causing Amyotrophic Lateral Sclerosis. the Winnower. Published 2017. Accessed.

5. Bennett JW, Klich M. Mycotoxins. Clin Microbiol Rev.2003;16(3):497-516.

6. Eskola M, Kos G, Elliott CT, Hajslova J, Mayar S, Krska R. Worldwide contamination of food-crops with mycotoxins: Validity of the widely cited 'FAO estimate' of 25. Crit Rev Food Sci Nutr. 2019:1-17.

7. Gruber-Dorninger C, Jenkins T, Schatzmayr G. Global Mycotoxin Occurrence in Feed: A Ten-Year Survey. Toxins (Basel).2019;11(7).

8. Auchtung TA, Fofanova TY, Stewart CJ, et al. Investigating Colonization of the Healthy Adult Gastrointestinal Tract by Fungi.mSphere. 2018;3(2).

9. de Hoog S, Monod M, Dawson T, Boekhout T, Mayser P, Gräser Y. Skin Fungi from Colonization to Infection. Microbiol Spectr.2017;5(4).

10. Al-Jaal BA, Jaganjac M, Barcaru A, Horvatovich P, Latiff A. Aflatoxin, fumonisin, ochratoxin, zearalenone and deoxynivalenol biomarkers in human biological fluids: A systematic literature review, 2001-2018. Food Chem Toxicol. 2019;129:211-228.

11. Omotayo OP, Omotayo AO, Mwanza M, Babalola OO. Prevalence of Mycotoxins and Their Consequences on Human Health. Toxicol Res.2019;35(1):1-7.

12. Dejardins AE. Fusarium Mycotoxins: Chemistry, Genetics and Biology. The American Phytopathological Society, St. Paul, Minnesota; 2006. 
13. Braun H, Buzina W, Freudenschuss K, Beham A, Stammberger H. 'Eosinophilic fungal rhinosinusitis': a common disorder in Europe? Laryngoscope. 2003;113(2):264-269.

14. Fungal Sinusitis. 2020.

15. deShazo RD, Chapin K, Swain RE. Fungal sinusitis. N Engl J Med. 1997;337(4):254-259.

16. Ponikau JU, Sherris DA, Kern EB, et al. The diagnosis and incidence of allergic fungal sinusitis. Mayo Clin Proc. 1999;74(9):877-884.

17. Waitzman AA, Birt BD. Fungal sinusitis. J Otolaryngol.1994;23(4):244-249.

18. Dai C, Xiao X, Sun F, et al. T-2 toxin neurotoxicity: role of oxidative stress and mitochondrial dysfunction. Arch Toxicol.2019;93(11):3041-3056.

19. Gabal MA, Awad YL, Morcos MB, Barakat AM, Malik G. Fusariotoxicoses of farm animals and mycotoxic leucoencephalomalacia of the equine associated with the finding of trichothecenes in feedstuffs. Vet Hum Toxicol. 1986;28(3):207-212.

20. Raymond SL, Smith TK, Swamy HV. Effects of feeding a blend of grains naturally contaminated with Fusarium mycotoxins on feed intake, metabolism, and indices of athletic performance of exercised horses. $J$ Anim Sci. 2005;83(6):1267-1273.

21. Riet-Correa F, Rivero R, Odriozola E, Adrien Mde L, Medeiros RM, Schild AL. Mycotoxicoses of ruminants and horses. $J$ Vet Diagn Invest. 2013;25(6):692-708.

22. Mishra S, Srivastava S, Dewangan J, Divakar A, Kumar Rath S. Global occurrence of deoxynivalenol in food commodities and exposure risk assessment in humans in the last decade: a survey. Crit Rev Food Sci Nutr. 2019:1-29.

23. Githang'a D, Anzala O, Mutegi C, Agweyu A. The effects of exposures to mycotoxins on immunity in children: A systematic review. Curr Probl Pediatr Adolesc Health Care. 2019;49(5):109-116.

24. Oswald IP, Marin DE, Bouhet S, Pinton P, Taranu I, Accensi F. Immunotoxicological risk of mycotoxins for domestic animals. Food Addit Contam. 2005;22(4):354-360.

25. Pestka JJ, Bondy GS. Alteration of immune function following dietary mycotoxin exposure. Can J Physiol Pharmacol.1990;68(7):1009-1016.

26. Reddy KE, Song J, Lee H-J, et al. Effects of High Levels of Deoxynivalenol and Zearalenone on Growth Performance, and Hematological and Immunological Parameters in Pigs. Toxins. 2018;10(3):114.

27. Ostermeyer-Shoaib B, Patten BM. IgG subclass deficiency in amyotrophic lateral sclerosis. Acta Neurol Scand.1993;87(3):192-194.

28. Beers DR, Zhao W, Wang J, et al. ALS patients' regulatory T lymphocytes are dysfunctional, and correlate with disease progression rate and severity. JCI Insight. 2017;2(5):e89530. 